\title{
Iatrogénie médicamenteuse, usage à domicile et innovation numérique : vers une révolution des métiers de la santé ?
}

\section{Drug-related iatrogenia, home use and digital innovation: towards a revolution of the health professions?}

\author{
G. Sacco • O. Guerin \\ C Springer-Verlag France 2014
}

Avec près de $20 \%$ des causes d'hospitalisation au-delà de 80 ans, la iatrogénie médicamenteuse reste une cause importante de morbidité et de mortalité dans la population gériatrique. Ainsi, plusieurs milliers de patients décèdent chaque année en France d'accidents iatrogéniques. C'est face à ce constat que depuis de nombreuses années, la gériatrie a fondé une partie de son action. En effet, face à une population de sujets âgés polypathologiques et donc polymédiqués, la hiérarchisation des priorités thérapeutiques à l'aide de la balance bénéfice-risque est indispensable. Pour ce faire, le gériatre met ainsi du côté risque de la balance la iatrogénie, et du côté bénéfice, non pas tant la morbidité ou la mortalité que la qualité de vie et le fonctionnement de l'individu dans son environnement. Contrairement à une pratique encore récente, cette hiérarchisation n'est pas systématiquement synonyme de suppression de molécules sur les ordonnances de nos patients. En effet, aux côtés de la notion d'overuse (sur-prescription) bien connue des gériatres depuis de nombreuses années, se sont développées les notions de misuse (mésusage) et underuse (sous-prescription). Cette dernière notion prend une place de plus en plus importante au fil du recul de l'âgisme dans les prises en charge. Ainsi, il n'est pas rare de voir les gériatres réintroduire ici un bêtabloquant chez un patient insuffisant cardiaque post-coronaropathie, ou là un anticoagulant dans un contexte de fibrillation auriculaire sous-traité par surévaluation du risque hémorragique. Mais c'est surtout sur le bon usage à domicile que se situent les enjeux de demain en termes de iatrogénie. Celui-ci passe par l'éducation thérapeutique des patients et de leurs aidants, ainsi que par l'utilisation de nouvelles solutions numériques d'aides à la dispensation et à l'observance. Ce nouveau pas ne se fera pas sans une évolution importante des métiers de la santé à laquelle il faut dès aujourd'hui se préparer.

\footnotetext{
G. Sacco $(\bowtie) \cdot$ O. Guerin

Pôle de gérontologie, $\mathrm{CHU}$ de Nice

e-mail : sacco.g@chu-nice.fr

G. Sacco

Centre Edmond et Lily Safra, Institut Claude Pompidou,

10 rue Molière, F-06100 Nice, France

O. Guerin

EA CoBTeK, Université de Nice Sophia-Antipolis
} 